\title{
Wi-Fi signal strengths database construction for indoor positioning systems using Wi-Fi RFID
}

\begin{abstract}
Nowadays, fingerprinting based $\mathrm{Wi}-\mathrm{Fi}$ positioning systems successfully provide location information to mobile users. Main idea behind fingerprinting is to build signal strength database of target area prior to location estimation. This process is called calibration. Indoor positioning system accuracy highly depends on calibration (sampling) intensity. This procedure requires huge amount of time and effort, and makes large-scale deployments of indoor positioning systems non-trivial. Newly constructed database may no longer be valid if there are any major changes in the target site. In this research we present a new approach of constructing fingerprint database. We propose a hybrid calibration procedure that combines signal sampling process with path-loss prediction algorithm. Instead of manual signal sampling, proposed method requires several Wi-Fi RFID tags to be installed in a target site. Advantage of such tag is that it can be read directly by commercial Wi-Fi access points from long distance. Several RFID tags mounted in target area will monitor the signal strength levels continuously and send scan data to the server. Whenever there are significant changes in signal levels detected, server will initiate database reconstruction procedure. Compared to existing calibration procedure our method requires only few signal samples from RFID tags to be collected and rest of the database is recovered using path-loss prediction algorithm.
\end{abstract}

Keyword: Indoor positioning; Wi-Fi ID; Path-loss prediction; RSSI 